\title{
CARACTERISTICAS DEL MAGMATISMO POST-OROGENICO FINIBRASILIANO EN EL URUGUAY: FORMACIONES SIERRA DE RÍOS Y SIERRA DE ANIMAS
}

\author{
JORGE BOSSI*, CARLOS CINGOLANI**, EDUARDO LAMBÍAS**, RICARDO VARELA** \\ y NÉSTOR CAMPAL*
}

\begin{abstract}
THE MAGMATIC CHARACTERISTICS OF THE POST-OROGENIC LATE BRAZILIAN MAGMATISM FROM URUGUAY: SIERRA DE RIOS AND SIERRA DE ANIMAS FORMATIONS. Two volcanic and sub-volcanic units from the Cuchilla Dionisio Belt assigned to the Late Brazilian Cycle in Uruguay, show $\mathrm{Rb}$-Sr isochrons indicativo of the Precambrian-Cambrian boundary, and the Cambrian. The Sierra de Rios Formation, yilded an age of $575 \pm 14 \mathrm{Ma}(\mathrm{Ri}=0.71046 \pm 0.00069)$, and the Sierra de Animas Formation an age of $520 \pm 5 \mathrm{Ma}(\mathrm{Ri}=0.70655 \pm 0.00010)$. The geochemical data of both units suggest that they originated during a post-orogenic crustal event, in which regional megafaulting was related to an exténsional rift environment.
\end{abstract}

Keywords: Brasiliano, post-orogenic magmatism, Rb-Sr geochronology, geochemistry, Uruguay.

\begin{abstract}
RESUMEN Se realizaron estudios petrográficos, geoquímicos y geocronológicos sobre rocas volcánicas y sub-volcanicas de dos unidades finibrasilianas del denominado Cinturón Cuchilla Dionisio en la R.O.Uruguay. $\mathrm{La}$ aplicación de isócronas Rb-Sr permitió definir edades en el deslinde Precámbrico-Cámbrico y Cámbrico: 1 . Formación Sierra de Ríos, $575 \pm 14 \mathrm{Ma}(\mathrm{Ri}=0,71046 \pm 0,0069)$; y 2. Formación Sierra de Animas, $520 \pm 5 \mathrm{Ma}$ $(\mathrm{Ri}=0,70655 \pm 0,00010)$. Las características de composición química son afines a rocas de origen cortical y sugieren vinculación a etapas distensivas post-orogénicas y a megatranscurrencias.
\end{abstract}

Palavras clave: Brasiliano, magmatismo post-orogenico, geocronologia Rb-Sr, geoquímica, Uruguay.

INTRODUCCIÓN Por relevamientos geológicos regionales y locales, ha sido reconocido, en Uruguay, un extenso y diseminado magmatismo efusivo e hipabisal, que se asocia a episodios distensivos de las etapas finales del Ciclo Brasiliano. Algunos de estos eventos fueron descriptos por Bossi \& Navarro (1991), mientras que otros se mencionan aqui por primera vez.

Vinculados a este amplio proceso cortical se incluyen provisoriamente las sienitas, microsienitas, traquitas, traquiandesitas, riolitas e ignimbritas que no muestran señales petrográficas de metamorfismo y atraviesan rocas del basamento precámbrico. En algunos casos, el grado de precisión estratigráfica aumenta (Formación Sierra de Ríos), porque estas rocas están cubiertas por sedimentitas glaciales pérmicas.

La eventual importancia de este magmatismo, con la posibilidad de interpretar las condiciones geotectónicas reinantes inmediatamente después de la aglutinación finicambriana de la porción occidental del paleocontinente de Gondwana, condujo a concretar en 1991, un convenio de cooperación científica entre el CONICET (Argentina) y la Universidad de la República (Uruguay), para llevar adelante el proyecto "Magmatismo Paleozoico asociado al Cratón del Río de la Plata".

El grado de conocimiento adquirido desde la fecha en que se iniciara este proyecto conjunto, ha permitido subdividir la porción uruguaya del Cratón del Río de la Plata (sensu Dalla Salda et al. 1988) en dos terrenos tectonoestratigráficos separados por un límite tectónico de primer orden (Bossi \& Campal 1992): Piedra Alta, al Oeste, y Nico Pérez, al Este. En el borde oriental de éste último se adosa el Cinturón Cuchilla Dionisio que constituyó una faja móvil durante el Neoproterozoico-Cámbrico (Ciclo Brasiliano) y que fuera descripto como Cinturón Dom Feliciano aplicando el modelo de evolución por tectónica de placas por Fragoso Cesar et al. (1982, 1987).

En la figura 1 se presenta un bosquejo general de la distribución de los terrenos citados, del magmatismo analizado en este trabajo y su relación geográfica preliminar, con las grandes unidades tectono-estratigráficas precámbricas del Uruguay.

El objetivo del trabajo es el de aportar nuevos datos de reconocimientos geológicos, estudios petrográficos, estructurales, geoquímicos y geocronológicos de dos unidades litoestratigráficas asociadas al Cinturón Cuchilla Dionisio. Se trata de las Formaciones Sierra de Ríos (Elizalde et al. 1970) y Sierra de Animas (Bossi 1966). Cabe destacar que éstas unidades fueron seleccionadas para su estudio en primer término porque poseían el mayor volumen de antecedentes geológicos. En este aspecto es de recordar que los trabajos geocronológicos de Hart (1966), Umpierre (1966), Umpierre \& Halpern (1971) y Halpern eí a/. (1972) han sido los primeros en destacar la presencia de un cinturón de rocas ígneas y metamórficas en el Sureste de Uruguay con edades K-Ar y Rb-Sr entre 500 y $600 \mathrm{Ma}$.

Para el presente trabajo los análisis químicos fueron realizados en el Laboratorio de Geoquímica del Centro de Investigaciones Geológicas (CIG) de la Facultad de Ciencias Naturales y Museo de la Universidad Nacional de La Plata. Se analizó el hierro como $\mathrm{Fe}_{2} \mathrm{O}_{3}$ total y se utilizó la fórmula de Le Maitre (1978) para su transformación parcial en $\mathrm{FeO}$.

Por su parte los datos geocronológicos se obtuvieron sobre roca total, a partir de muestras seleccionadas según su contenido total en $\mathrm{Sr}$ y $\mathrm{Rb}$ por FRX (error menor a $2 \%$ ). El ataque químico y la concentración de estroncio natural 
se realizaron en el Laboratorio $\mathrm{Rb}-\mathrm{Sr}$ del CIG, La Plata, y la espectrometría de masas, en el Centro de Pesquisas Geocronológicas (CPGeo) de la Universidad de Sao Paulo, Brasil.

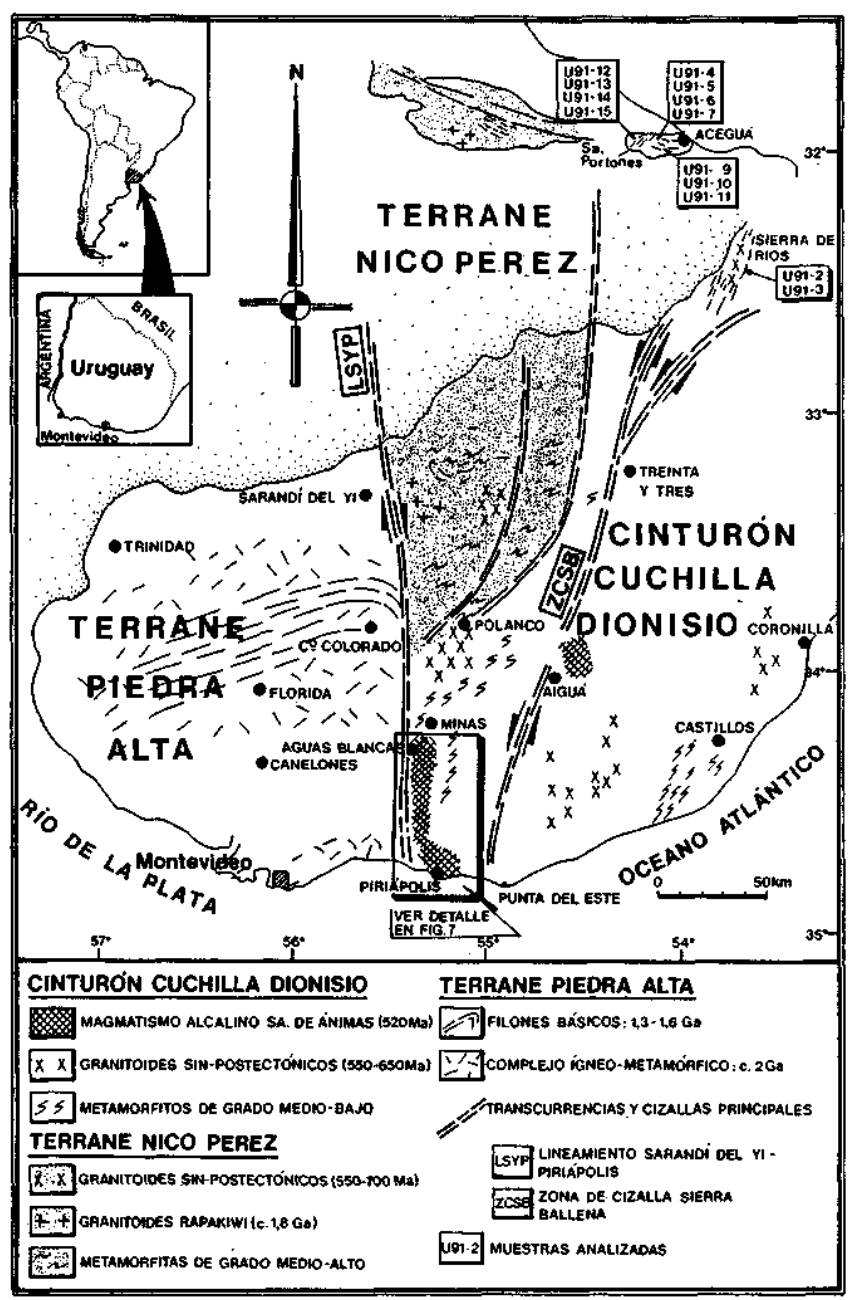

Figura 1 -Bosquejo tectónico regional y ubicación de la zona de estudio. Modificado de Bossi \& Campal (1992) Figure 1 - Regional tectonic sketch map and location of the studied área (modified from Bossi \& Campal 1992)

LA FORMACIÓN SIERRA DE RÍOS Los afloramientos estudiados se encuentran en el sector Nordeste de Uruguay, en los alrededores de la localidad de Aceguá, en la Sierra de Ríos y Sierra de Portones (véase Fig. 1).

Esta unidad litoestratigráfica fue separada por Elizalde et al. (1970), como un conjunto de filones y derrames riolíticos, con una posición estratigráfica imprecisa, enmarcada entre el Cámbrico y el final del Carbonífero. Es de señalar que intrusivas acidas subvolcánicas (microgranitos) fueron estudiadas también por Cordani \& Soliani (1990) en el Norte de Uruguay, Departamento de Rivera. La continuación en la porción sudoeste del Escudo de Río Grande do Sul (Brasil) fue analizada también por Nardi et al. (1992), quienes destacaron la presencia de un intenso magmatismo no deformado de edad eopaleozoica, de afinidades shoshonítica y alcalina. Por su parte, Sommer et al. (1993) definieron un vulcanismo acidó alcalino en el denominado "plateau de Tacuarembó" para la región de Dom Pedrito, RS. Según los autores mencionados los datos petrográficos y geoquímicos sugieren un magmatismo de naturaleza alcalina o peralcalina que puede estar asociado a las últimas manifestaciones de la orogenia brasiliana.

Petrografía Los filones de riolitas, de rumbo dominante $\mathrm{N} 5 \mathrm{OE}$, atraviesan rocas graníticas pertenecientes al Ciclo Brasiliano y son cubiertos por sedimentos gondwánicos. Estas riolitas son siempre porfiricas con fenocristales de cuarzo y feldespatos, que normalmente es ortosa pertítica. Son frecuentes los intercrecimientos eutécticos cuarzo-ortosa. La matriz es granofírica. Los filones estudiados corresponden a típicos granófiros riolíticos de matriz afanítica y dos microgranitos.

Los microgranitos en diques de 10 - $20 \mathrm{~m}$ de espesor poseen textura afanítica en contacto con las encajantes. Sus constituyentes minerales son: ortosa micropertítica, automorfa a subautomorfa; plagioclasa en dos generaciones, la primera zoneada, automorfa, se corresponde con una oligoclasa media y la segunda de bordes sinuosos algo más acida muestra muy abundantes mirmequitas en todos los individuos. El único máfico no opaco presente en cantidad significativa es la biotita, en proporción menor al $5 \%$.

Los derrames conservan rasgos ignimbríticos y contienen hasta un $40 \%$ de fenocristales de cuarzo, ortosa y albita. La matriz es afanítica criptocristalina. Estos derrames riolíticos se apoyan sobre un zócalo granítico peneplanizado, con plano de discordancia subhorizontal, que no ha sido deformado ni sometido a importantes ascensos con posterioridad a su efusión, confirmando su carácter de magmatismo post-orogénico.

Características químicas Los datos químicos logrados sobre 13 muestras, que se exponen en la tabla I, revelan la presencia de una asociación ígnea de alta sílice. Las rocas volcánicas se clasifican como riolitas en el diagrama TAS y los microgranitos y granitos presentan la misma composición, por lo cual se comprueba que forman parte de la misma asociación magmática (Fig. 2). Las rocas se caracterizan por su alto contenido en potasio (Fig. $3 ; \mathrm{K}_{2} \mathrm{O} / \mathrm{Na}_{2} \mathrm{O}$ entre 1,0 y 2,1), por una débil peraluminosidad (Fig. 4; A/ CNK entre 1,0 y 1,2 ) y relativamente bajo calcio, siendo las ignimbritas las que mayor cantidad poseen (Fig. 5).Estas concentraciones indican una cierta componente cortical en la evolución del magma, que es confirmada por las altas relaciones isotópicas iniciales $\mathrm{Sr}^{87} / \mathrm{Sr}^{86}$ (c/. infra).

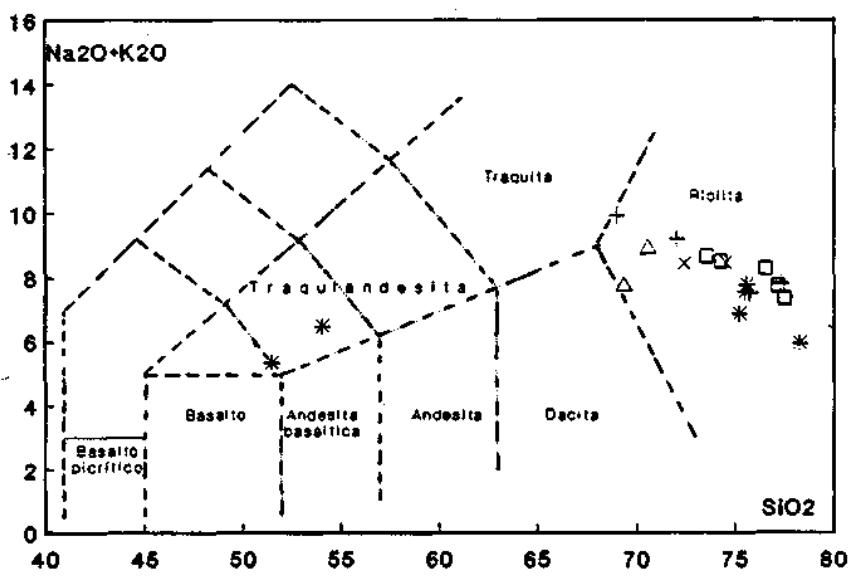

Figura 2 - Diagrama álcalis vs. sílice, base anhidra, con la nomenclatura de las volcanitas, según Le Maitre (1989). Los símbolos son los mismos que los de la figura 5 Figure 2 - TAS diagram for studied samples in anhydrous base. Classification of the volcanics according to Le Maitre (1989). Same symbols as figure 5 


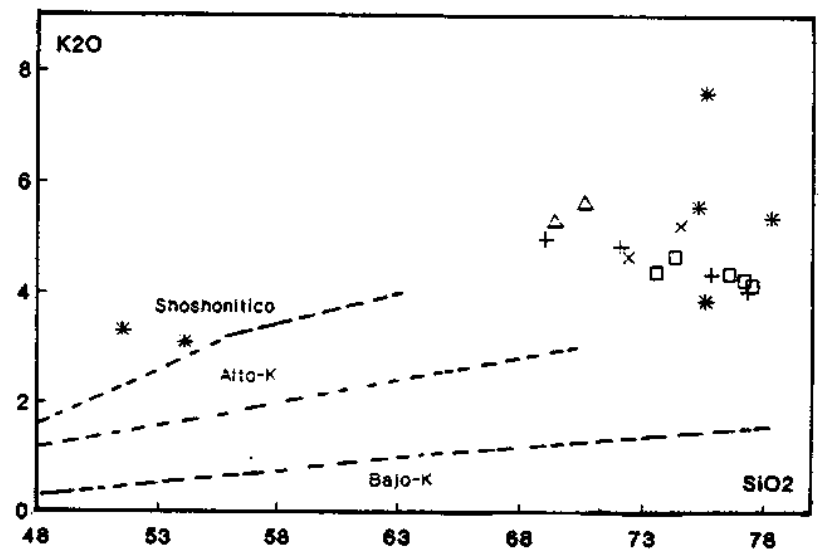

Figura 3 - Diagrama $\mathrm{K}_{2} \mathrm{O}$ vs. $\mathrm{SiO}_{2}$, base anhidra, con la clasificación de las vulcanitas de acuerdo al contenido de potasio según Peccerillo \& Taylor (1976). Los símbolos son los mismos que los de la figura 5

Figure $3-\mathrm{K}_{2} \mathrm{O}$ vi. $\mathrm{SiO}_{2}$ diagram anhydrous base. Classification of volcanics according to Peccerillo \& Taylor (1976). Symbols as in figure 5

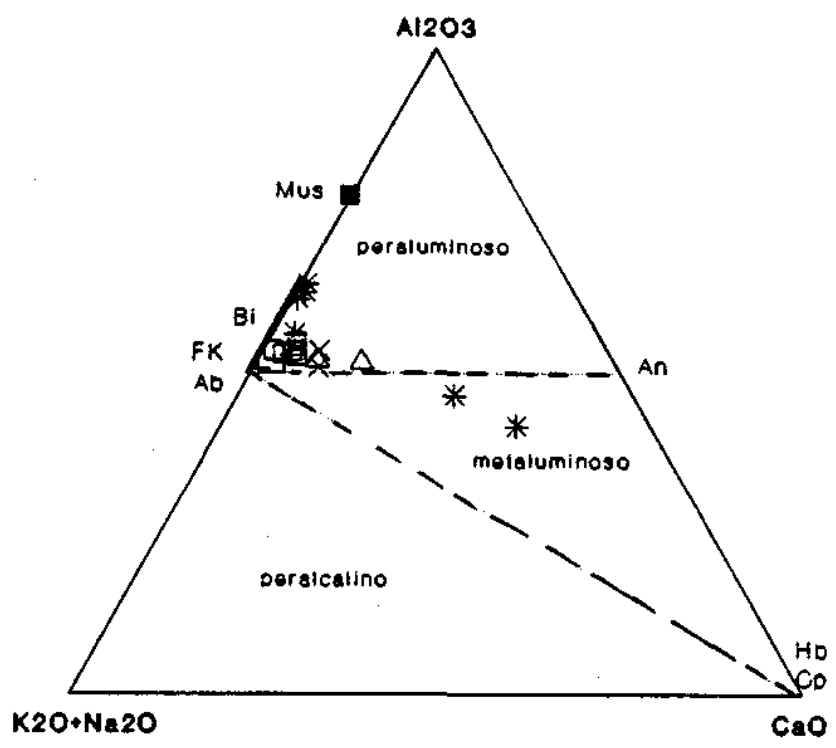

Figura 4 - Diagrama $\mathrm{Al}_{2} \mathrm{O}_{3}-\mathrm{K}_{2} \mathrm{O}+\mathrm{Na}_{2} \mathrm{O}-\mathrm{CaO}$ molar de las rocas estudiadas. Los campos de saturación en alúmina están basados en Patiño-Douce (1992). Ab = Albita, An = Anortita, Bi - Biotita, $C p=$ Clinopiroxeno, $F K=$ Feldespato potásico, $\mathrm{Hb}=$ Hornblenda, Mus - Muscovita. Los símbolos son los mismos que los de la figura 5 Figure $4-\mathrm{Al}_{2} \mathrm{O}_{3}-\mathrm{K}_{2} \mathrm{O}+\mathrm{Na}_{2} \mathrm{O}-\mathrm{CaO}$ molar diagram. Alumina saturation división according to Patiño-Douce (1992). Ab = albite, $\mathrm{An}=$ anorthite, $\mathrm{Bi}$ $=$ biotite, $\mathrm{Cp}=$ clinopyroxene, $\mathrm{FK}=\mathrm{K}$-feldspar, $\mathrm{Hb}=$ hornblende, Mus = muscovite. Symbols as in figure 5.

Geocronología Un antecedente para el área de las denominadas "Islas Cristalinas" de Rivera y Aceguá, es el trabajo efectuado por Cordani \& Soliani (1990). Considerado por los citados investigadores un reconocimiento geocronológico preliminar, brinda edades $\mathrm{K}-\mathrm{Ar}$ y $\mathrm{Rb}-\mathrm{Sr}$ para rocas metamórficas del Basamento Transamazónico (Proterozoico inferior, $2204 \pm 65 \mathrm{Ma}$ ), granitoides porfiroides sintectónicos vinculables al Ciclo Brasiliano (Proterozoico Superior, $680 \pm 22 \mathrm{Ma}$ ), granitos brasilianos tardi a

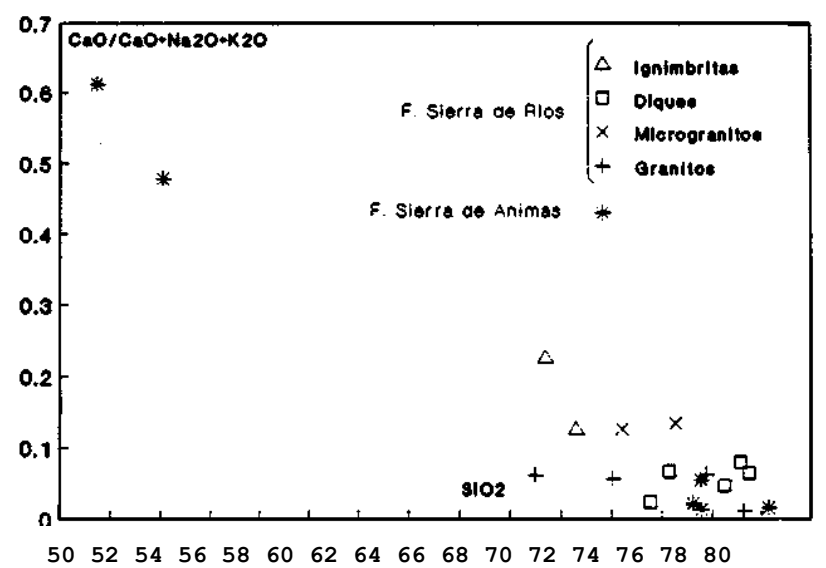

Figura 5 - Diagrama $\mathrm{CaO} / \mathrm{CaO}+\mathrm{Na}_{2} \mathrm{O}+\mathrm{K}_{2} \mathrm{O}$ vs. $\mathrm{SiO}_{2}$ exponiendo el bajo contenido de calcio en las rocas de alta silice

Figure $5-\mathrm{CaO} / \mathrm{CaO}+\mathrm{Na}_{2} \mathrm{O}+\mathrm{K}_{2} \mathrm{O}$ vs. $\mathrm{SiO}_{2}$ diagram, showing the low calcium contení for high silica samples

post-tectónicos (límite Precámbrico-Paleozoico, 570 - 580 Ma). Microgranitos de la Isla Rivera fueron datados por el método K-Ar con valores entre 520-540 Ma e indicados como anorogénicos.

En la presente contribución se seleccionaron y procesaron cuatro muestras de roca total (véase Fig. 1), correspondientes a dos microgranitos (U-91-5 y U-91-7) y dos ignimbritas (U-91-2 y U-91-3), que definen una recta de $575 \pm 14 \mathrm{Ma}$ (1 sigma) con $\mathrm{Ri}=0,71046 \pm 0,00069$ (véase Tab. II y Fig. 6).

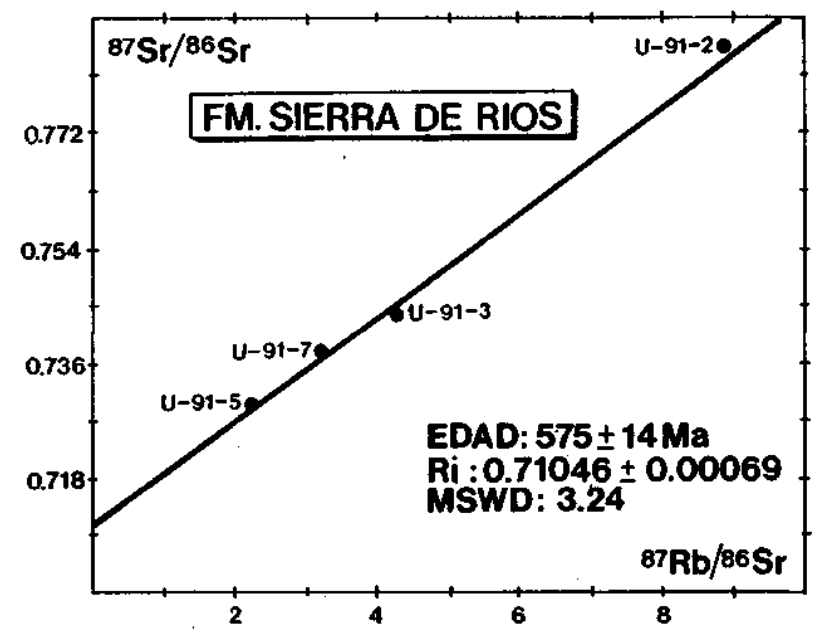

Figura 6 - Diagrama de evolución isotópica $R b-S r$ de muestras de la Formación Sierra de Ríos. $R b^{87}: 1,42$ $x 10^{-11} / a$

Figure 6 - Strontium isotopic evolution diagram for samples of Sierra de Ríos Formation. $\mathrm{Rb}^{87}: 1.42 \times 10^{-11} / \mathrm{a}$

La edad obtenida ubica al magmatismo de la Formación Sierra de Ríos, al menos para la zona muestreada, en el deslinde Precámbrico-Cámbrico, indicando fuerte ascenso del granito encajante, cristalizado a varios kilómetros de profundidad. 
Tabla 1 - Datos correspondientes a análisis químicos de muestras de las Formaciones Sierra de Ríos y Sierra de Animas Table I - Chemical compositions of samples from Sierra de RíoS and Sierra de Animas Formations

\begin{tabular}{|c|c|c|c|c|c|c|c|c|c|c|c|c|c|c|c|c|c|c|c|}
\hline \multirow[b]{4}{*}{ Muestra $\%$} & \multicolumn{13}{|c|}{ Formación Sierra de Rios } & \multirow{2}{*}{\multicolumn{6}{|c|}{$\begin{array}{l}\text { Formaciont } \\
\text { Sierra de Animas }\end{array}$}} \\
\hline & \multicolumn{9}{|c|}{ Acegua - Sterra de Rios } & \multirow{2}{*}{\multicolumn{4}{|c|}{$\frac{\text { Sierra Pottones }}{\text { Gramilos }}$}} & & & & & & \\
\hline & \multicolumn{2}{|c|}{ Igrimbrita } & \multicolumn{5}{|c|}{ Digues } & \multicolumn{2}{|c|}{ Microgranitos } & & & & & \multicolumn{4}{|c|}{ Riolitas } & \multicolumn{2}{|c|}{ Traquiloides } \\
\hline & U91-2 & U9]-3 & U91.4 & U91-6 & U91.9 & U9:1-10 & U91-11 & U91-5 & U91-7 & U91-12 & U91-13 & U91-14 & U91-15 & U9].17 & U91.18 & U91.19 & U91.20 & U91-21 & U91-22 \\
\hline $\mathrm{SiO}_{2}$ & 68,30 & 69,40 & 73,00 & 76,60 & 76,80 & 73,50 & 76,08 & 74,20 & 71,85 & 75,50 & 71,55 & 68,50 & 77,00 & 74,50 & 77,40 & 74,37 & 74,52 & 50,90 & 50,00 \\
\hline $\mathrm{TiO}_{2}$ & 0,44 & 0,52 & 0,32 & 0,08 & 0,10 & 0,18 & 0,12 & 0.19 & 0,30 & 0,13 & $0, \mathbf{3 3}$ & 0,47 & 0,23 & 0,38 & 0,08 & 0,30 & 0,13 & 0,19 & 1,44 \\
\hline $\mathrm{Al}_{2} \mathrm{O}$ & 14,30 & 14,35 & 13,53 & 12,40 & 12,50 & 13,80 & 13,00 & 13,40 & 14,91 & 11,80 & 14,05 & 15,33 & 10,80 & 13,34 & 11,80 & 13,58 & 13,50 & 15,80 & 15,20 \\
\hline $\mathrm{Fe}_{2} \mathrm{O}$, & 4,26 & 3,10 & 2,95 & 1,44 & 1,42 & 1,97 & 1,46 & 1,46 & 1,95 & 1,80 & 2,99 & 3,88 & 4,08 & 2,01 & 3,02 & 2,89 & 1,87 & 10,80 & 11,80 \\
\hline Mno & 0.14 & 0,05 & 0,02 & 0,02 & 0,01 & 0,03 & 0,02 & 0,03 & 0,04 & 0,03 & 0,07 & 0,16 & 0,06 & 0,01 & 0,01 & 0,05 & 0,03 & 0,15 & 0,15 \\
\hline $\mathrm{MgO}$ & 0,24 & 0.31 & 0,20 & 0,01 & 0,01 & 0,14 & 0,01 & 0,24 & 0,39 & 0,01 & 0,24 & 0.10 & 0,01 & 0.14 & 0,01 & 0,14 & 0,15 & 4.68 & 4,66 \\
\hline $\mathrm{CaO}$ & 2,22 & 1,25 & 0,20 & 0,66 & 0,50 & 0,60 & 0,40 & 1,30 & 1,20 & 0.50 & 0,55 & 0.65 & 0.09 & 0,10 & 0,09 & 0,15 & 0,43 & 5,60 & 8,20 \\
\hline $\mathrm{Na}_{2} \mathrm{O}$ & 2.46 & 3,25 & 4,26 & 3,48 & 3,18 & 3,82 & 3,92 & 3,22 & 3,76 & 3,18 & 4,35 & 4,95 & 3,80 & 0,20 & 0,58 & 1,30 & 3,66 & 3.20 & 2,00 \\
\hline $\mathrm{K}_{2} \mathrm{O}$ & 5,20 & 5,52 & 4,34 & 4,20 & 4,10 & 4,60 & 4,32 & 5,20 & 4,60 & 4,32 & 4,80 & 4,93 & 4,01 & 7,49 & 3,30 & 3,50 & 3,80 & 2,91 & 3,20 \\
\hline $\mathrm{P}_{1} \mathrm{O}_{3}$ & 0,31 & 0,14 & 0,03 & 0,05 & 0,05 & 0,03 & 0,05 & 0,05 & 0,06 & 0,05 & 0,06 & 0,06 & 0,06 & 0,04 & 0,08 & 0,04 & 0,03 & 0,09 & 0,87 \\
\hline $\mathrm{H}_{2} \mathrm{O}^{+}$ & 1,18 & 1,40 & 0,50 & 0,52 & 0,70 & 0,73 & 0,38 & 0,28 & 0,50 & 0,28 & 0,33 & 0,50 & 0,30 & 1,28 & 0,78 & 0,90 & 1,10 & 5,33 & 2,58 \\
\hline $\mathrm{H}_{2} \mathrm{O}^{-}$ & 0,32 & 0,25 & 0,20 & 0,20 & 0,18 & 0,30 & 0,20 & 0,15 & $0,2.5$ & 0,05 & 0,35 & 0,20 & 0,10 & 0,13 & 0,33 & 0,25 & 0,22 & 0.53 & 0,30 \\
\hline Total & 99,37 & 99,54 & 99,55 & 99,66 & 99,55 & 99.70 & 99,96 & 99,72 & 99,81 & 97,65 & 99,67 & 99,73 & 100,54 & 99,62 & 99,48 & 99,47 & 99,44 & 100,18 & 100,40 \\
\hline Rb(ppm) & 193 & 192 & 199 & & & 182 & & 168 & 227 & & & 116 & & 314 & 224 & 215 & 181 & 53 & \\
\hline Sr(ppm) & 75 & 146 & 20 & & & 22 & & 218 & 230 & & & 34 & & 47 & 36 & 43 & 80 & 777 & \\
\hline $\operatorname{Zr}(\mathrm{ppm})$ & & 465 & 750 & & & 174 & & & 150 & & 206 & 166 & & & & 1500 & 210 & & \\
\hline $\mathrm{Nb}(\mathrm{ppm})$ & & 20 & 57 & & & 31 & & & 13 & & 21 & 144 & & & & 70 & 79 & & \\
\hline
\end{tabular}

Los enjambres de diques sugieren proceso extensional típico de etapas post-orogénicas. Es de señalar que en la región de Río Grande do Sul (Brasil) pequeños stocks de granitos pertíticos con riebeckita fueron ubicados en el Cámbrico mediante una edad convencional $\mathrm{Rb}-\mathrm{Sr}$ de $557 \pm 6 \mathrm{Ma}$ (W. Teixeira in Horbach et al 1986).

LA FORMACIÓN SIERRA DE ANIMAS Los afloramientos estudiados se encuentran en el sector centro-sur de Uruguay, entre las localidades de Pan de Azúcar y Piriápolis (véase Figs. 1,7 y 8).

La Formación Sierra de Animas fue instituida por Bossi (1966) para identificar una asociación de rocas volcánicas y subvolcánicas, principalmente mesosilícicas constituyendo un importante filón de rumbo general Norte-Sur, con $50 \mathrm{~km}$ de longitud y unos 4 a $6 \mathrm{~km}$ de ancho. Correspondieron a Bossi \& Fernandez (1963) los primeros aportes sobre descripciones petrográficas para esta formación.

Posteriormente, Bossi \& Navarro (1991) recompilaron la información existente hasta 1988, sobre cartografía geológica y petrografía de la unidad, compuesta por sienitas más o menos cuarzosas, traquitas, basaltos, granófiros y riolitas (Fig. 2). Simultáneamente, Preciozzi et al.(1991) publicaron la Carta Geológica del Uruguay 1:100.000, fotoplano Piriápolis, que incluye el extremo sur de la Formación, con descripciones petrográficas coincidentes (Fig. 8).

Analizando estos antecedentes a la luz de los datos geotectónicos actualmente disponibles, ha sido postulado que la geometría del magmatismo resulta de su desarrollo en una fosa tectónica con posteriores movimientos de basculación que generaron hundimientos relativos hacia el Norte y el Oeste.

En el tramo principal de su desarrollo (entre las localidades de Aguas Blancas y Pan de Azúcar), las rocas dominantes son traquitas en el Oeste y sienitas en el Este, donde la erosión expone niveles más profundos. En los alrededores de Piriápolis, las rocas más abundantes son sienitas de grano grueso y sienitas cuarzosas. El magmatismo comienza por efusión de traquiandesitas y queda limitado a una faja entre fallas de rumbo Noroeste, de donde se infiere su vinculación con el desarrollo de una fosa tectónica. En un sector de la misma, fueron observadas sedimentitas clásticas intercaladas con las coladas de materiales volcánicos.

Petrografía Las traquiandesitas fueron descriptas por Walther (1927) como rocas amigdaloides de textura traquítica y composición de andesitas (con andesina y diópsido). Preciozzi et al. (1991), por su parte, las describen como rocas volcánicas con vacuolas esféricas o estiradas rellenas de clorita, cuarzo y epídoto. Presentan evidencias de fuerte alteración hidrotermal, con formación de clorita, epidoto y carbonato. Para la realización del presente trabajo se muestrearon afloramientos libres de alteración superficial, para lograr definición geoquímica por elementos mayoritarios, su naturaleza petrográfica original y datación radimétrica. De acuerdo a los estudios petrográficos, fueron definidas traquiandesitas con típica textura traquítica con 50-60\% de bastones de andesina, 5-10\% de olivina automorfa, algo serpentinizada y abundante mesostasis vitrea.

Las sienitas afloran en la falda oriental de la Sierra de las Animas y como un extenso dique anular alrededor del cerro Pan de Azúcar. Son de textura granuda, hipidiomórfica, con cristales siempre mayores a $2 \mathrm{~mm}$. La mineralogía es relativamente constante: albita-oligoclasa, 45 - 55\%; ortosa pertítica, 15 - 25\%; micropegmatita, $10-20 \%$; hornblenda, 8 - 20\%; y arfvedsonita, excepcional.

Las microsienitas son facies frecuentes como filones y pequeños cuerpos ovoides. Tienen textura bostonítica y su 


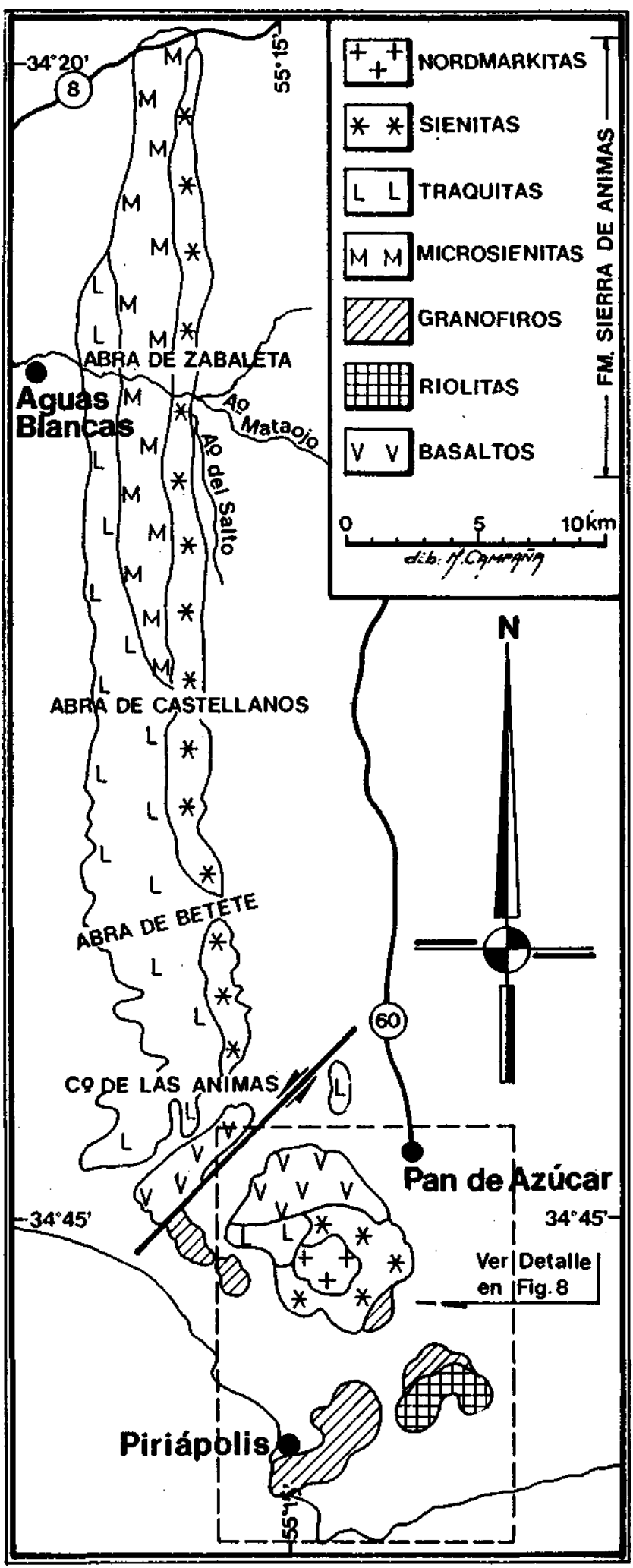

Figura 7 - Geología del sector de Sierra de Animas, basado en Bossi \& Navarro (1991)

Figure 7 - Geological sketch map of the Sierra de Animas región, according to Bossi \& Navarro (1991)

mineralogía contiene $85 \%$ de feldespato, $10 \%$ anfibol y $5 \%$ de cuarzo (Bossi \& Fernandez 1963).

Finalmente, las traquitas-traquiandesitas deben mencionarse como los tipos litológicos más abundantes de la unidad estudiada. Se trata en realidad de pórfidos afaníticos con fenocristales de ortosa de 3 a $5 \mathrm{~mm}$ de longitud. Las riolitas y pórfidos riolíticos tienen típica textura granofirica y micropegmatítica, con esporádicos fenocristales de sanidina. Es normal la presencia de cristales automorfos de circón.

Características químicas En virtud del porcentaje de vidrio presente en las rocas volcánicas integrantes de la Formación Sierra de Animas, se consideró imprescindible incluir análisis químicos para definir las características del magmatismo, que hasta el momento no poseía información. Los muéstreos realizados sobre afloramientos libres de alteración superficial o hidrotermal permitieron obtener especímenes frescos de cuatro granófiros riolíticos (U-9117, U-91-18, U-91-19 y U-91-20) y dos traquiandesitas (U-91-21 y U-91-22). Los resultados obtenidos se exponen en la tabla I.

La Formación Sierra de Animas en el sector de estudio está constituida por rocas de alta sílice y baja sílice, probablemente en una relación de tipo bimodal. Por su parte, las características químicas de las rocas de alta sílice son similares a las de la Formación Sierra de Ríos (alto potasio; $\mathrm{A} / \mathrm{CNK}$ entre 1,2 y 1,7 ; y bajo calcio). Las rocas de baja sílice, reconocidas en el campo como traquitoides, tienen alto contenido de calcio, y por la abundancia de álcalis muestran afinidades alcalinas, que por su elevada concentración de potasio tienen características shoshoníticas (véase Fig. 2,3,4y5)

La edad relativa de los traquitoides parece ser en algunos casos más antigua que la de las riolitas, como por ejemplo en Playa Hermosa, al este de Piriápolis, donde son cortados por diques de riolita. Sin embargo, ésta relación puede ser local y desprovista de significado geocronológico, por lo

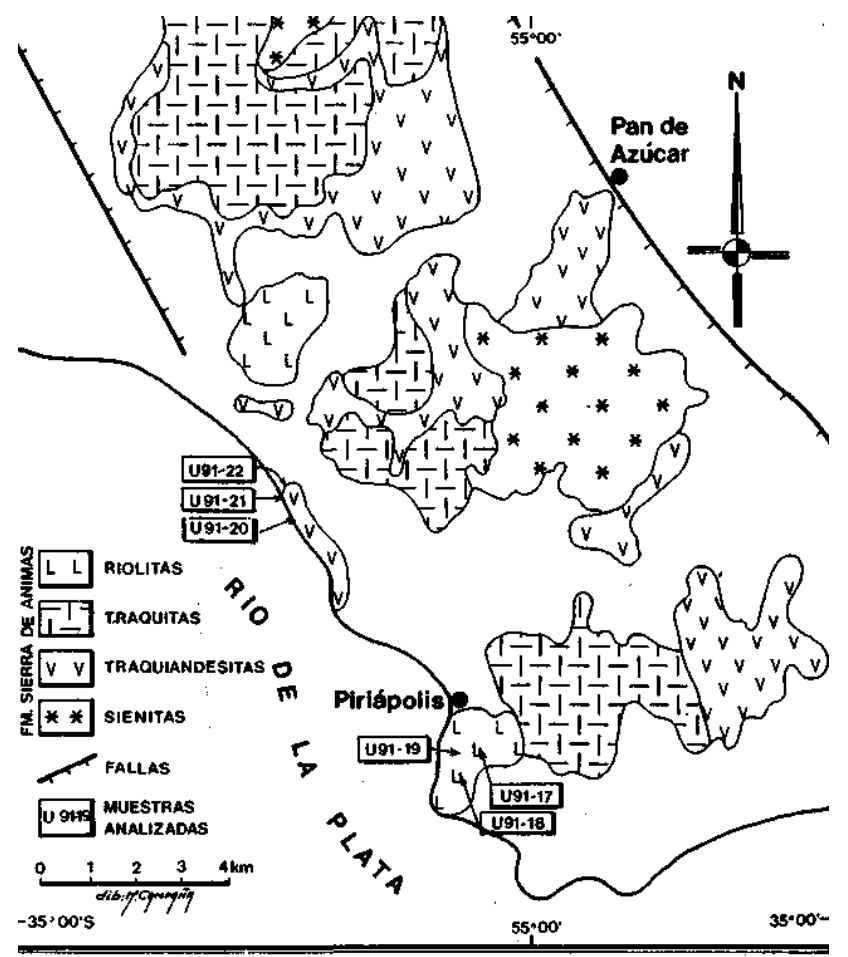

Figura 8- Geología del sector Pan de AzúcarPiriápolis, según Preciozzi et al. (1991). Ubicación de las muestras estudiadas

Figure 8 - Geology of the Pan de Azúcar - Piriápolis area, according to Preciozzi et al. (1991), with sample location 
Tabla II - Datos isotópicos de Rb-Sr de muestras de la Formación Sierra de Ríos

Table II - Rb-Sr isotopic data for samples of the Sierra de Ríos Formation

\begin{tabular}{|l|c|c|c|c|c|c|}
\hline \multicolumn{2}{|c|}{ MUESTRA } & & & & & \\
\cline { 1 - 2 } $\mathrm{N}^{2}$ Lab. & $\mathrm{n}^{\mathrm{Q}}$ Campo & Roca & Rb(ppm) & Sr(ppm) & $87 \mathrm{Rb} / 86 \mathrm{Sr}$ & $87 \mathrm{Sr} / 86 \mathrm{Sr}$ \\
\hline CIG 548 & U-91-2 & IGNIMBRITA & 190,00 & 63,00 & $8.7960 \pm 0.1760$ & $0.783980 \pm 0.000160$ \\
\hline CIG 549 & U-91-3 & IGNIMBRITA & 187,00 & 129,00 & $4.2112 \pm 0.0842$ & $0.743430 \pm 0.000100$ \\
\hline CIG 550 & U-91-5 & MICROGRANITO & 168,00 & 218,00 & $2.2356 \pm 0.0448$ & $0.728980 \pm 0.000070$ \\
\hline CIG 551 & U-91-7 & MICROGRANTTO & 216,00 & 195,00 & $3.2159 \pm 0.0644$ & $0.737120 \pm 0.000090$ \\
\hline
\end{tabular}

Tabla III - Datos isotópicos Rb-Sr de muestras de la Formación Sierra de Animas

Table III - Rb-Sr isotopic data for samples of the Sierra de Animas Formation

\begin{tabular}{|l|c|c|c|c|c|c|}
\hline \multicolumn{1}{|c|}{ MUESTRA } & & & & \\
\cline { 1 - 2 } N $^{2}$ Lab. & $N^{9}$ Campo & Roca & Rb(ppm) & Sr(ppm) & $87 \mathrm{Rb} / 86 \mathrm{Sr}$ & $87 \mathrm{Sr} / 86 \mathrm{Sr}$ \\
\hline CIG 555 & U-91-17 & RIOLITA & 324,00 & 29,00 & $33.0870 \pm 0.6600$ & $0.942620 \pm 0.000120$ \\
\hline CIG 556 & U-91-18 & RIOLITA & 224,00 & 36,00 & $18.2560 \pm 0.3600$ & $0.845410 \pm 0.000810$ \\
\hline CIG 557 & U-91-20 & RIOLITA & 166,00 & 58,00 & $8.3360 \pm 0.1660$ & $0.769930 \pm 0.000090$ \\
\hline CIG 558 & U-91-21 & TRAQUITOIDE & 53,00 & 777,00 & $0.1970 \pm 0.0040$ & $0.708000 \pm 0.000090$ \\
\hline CIG 560 & U-91-19 & RIOLITA & 222,00 & 29,00 & $22.5160 \pm 0.4500$ & $0.871290 \pm 0.000210$ \\
\hline
\end{tabular}

cual su generalización a toda la región no es posible con los datos que se poseen.

Geocronología Esta unidad estratigrafía, cuya posición cronológica era dudosa, pensándose incluso que fuera un magmatismo mesozoico, fue objeto de estudios geocronológicos preliminares, por el método K-Ar, en el Centro de Pesquisas Geocronológicas de la Universidad de Sao Paulo (Umpierre 1966). Los datos obtenidos por esta autora son los siguientes:

\begin{tabular}{|l|l|l|l|}
\hline Localidad & Roca & Datacion sobre & Edad (Ma) \\
\hline Aguas Blancas & Traquita & Roca total & 508 \\
\hline C. San Antonio & Gran6firo & Roca total & 519 \\
\hline Piriápolis & Sienita Qz. & Feldspato potásico & 487 \\
\hline Piriápolis & Sienita Qz. & Anfibol & 552 \\
\hline
\end{tabular}

Estas edades K-Ar fueron valiosas para ubicar en el Paleozoico inferior al magmatismo de referencia. Posteriormente, Soliani et al. (1984) dieron a conocer una síntesis geocronológica de la porción meridional del escudo atlántico. En forma reciente, Preciozzi et al. (1993) aportaron nuevos datos geoquímicos y geocronológicos sobre el magmatismo tardi a post-transcurrente de Uruguay. Incluyen una isócrona $\mathrm{Rb}-\mathrm{Sr}$ de $499 \pm 72 \mathrm{Ma}$, con $\mathrm{Ri}=0.7063$ para los sienogranitos de Las Animas, emplazados en ambientes distensivos.

Con el objeto de ampliar ésta información, se programó el estudio por el método $\mathrm{Rb}-\mathrm{Sr}$ sobre roca total, a partir de litologías cogenéticas y adecuadamente dispersas en su contenido isotópico. Los resultados analíticos se presentan en la tabla III y figura 9.

Se. obtuvieron datos isotópicos (roca total) sobre cinco muestras de vulcanitas, quatro de ellas de composición riolítica (U-91-17, U-91-18, U-91-19, U-91-20) y una traquita (U-91-21), con una muy amplia variedad composicional $\left(\mathrm{Rb}^{87} / \mathrm{Sr}^{86}\right.$ de hasta 33,1$)$, que arrojan una edad de $520 \pm 5 \mathrm{Ma}$ (1 sigma). La relación inicial es de $0.70655 \pm$ 0.00010 (1 sigma).

Esta edad geocronológica $\mathrm{Rb}-\mathrm{Sr}$ (similar en el caso con la obtenida por Umpierre 1966, por el método K-Ar en

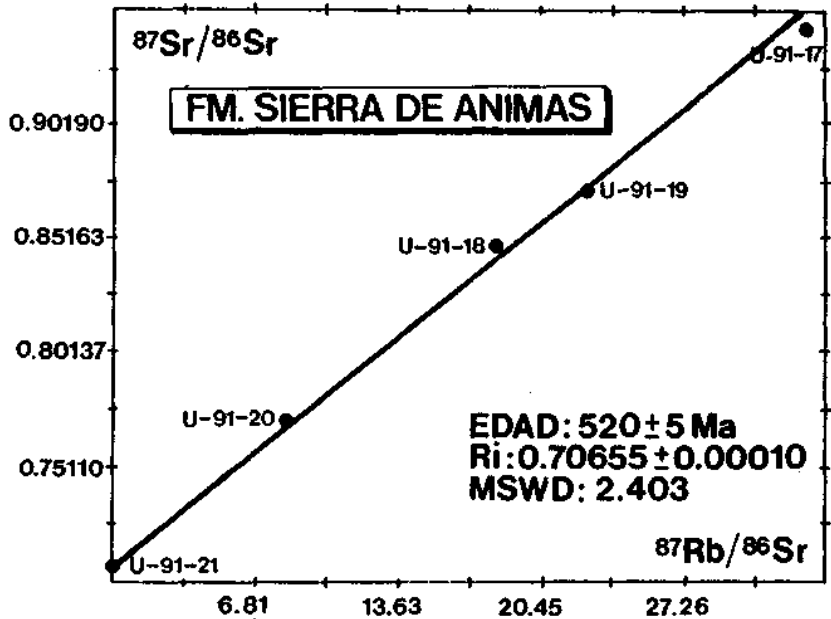

Figura 9 - Diagrama de evolución isotópica $\mathrm{Rb}-\mathrm{Sr}$ de muestras de la Formación Sierra de Animas. $R b^{87}: 1.42 x$ $10^{-11} / a$

Figure 9 - $\mathrm{Rb}-\mathrm{Sr}$ isotopic evolution diagram for samples of the Sierra de Animas Formation. $\mathrm{Rb}^{87}: 1.42 \times 10^{-11} / \mathrm{a}$

granófiros del Cerro San Antonio) resulta de apreciable valor estratigráfico, porque tiene coherencia con la edad geológica asignada a este magmatismo en fase distensiva como inmediatamente posterior a la transcurrencia Sarandi del Yi-Piriápolis, que fuera datada por el método $\mathrm{Rb}-\mathrm{Sr}$ en roca total en $530 \pm 15 \mathrm{Ma}$ (Bossi \& Campal 1992).

CONCLUSIONES Se estudiaron aspectos petrográficos, geoquímicos y geocronológicos de dos magmatismos vinculados a períodos distensivos finibrasilianos. Las asociaciones litológicas y la edad de emplazamiento de cada una son diferentes: la Formación Sierra de Ríos, integrada exclusivamente por rocas acidas (riolitas-granitos), tuvo emplazamiento a los $575 \pm 14$ Ma de antigüedad; la Formación Sierra de Animas, incluye rocas mesosilícicas (traquitas y traquiandesitas) y acidas (riolitas), ubicándose 
con edad de $520 \pm 5$ Ma. Estos dos eventos magmáticos reflejan distintas y sucesivas etapas de un estadio postorogénico brasiliano, en el que se distingue uno temprano, caracterizado por alta sílice (Fm. Sierra de Ríos) y otro tardío de tipo bimodal con rocas alcalinas y de alta sílice (Fm. Sierra de Animas).

La asociación de alta sílice de la Formación Sierra de Ríos es característica de ambientes post-orogénicos asociados a levantamiento cortical. La superficie de erosión, que constituye casi una peneplanicie sobre la cual fluyeron las ignimbritas, indica fuerte ascenso y erosión, mientras que los enjambres de diques silícicos sugieren período de extensión probablemente relacionado al desmoronamiento de una corteza engrosada como consecuencia de una orogénesis previa. En la Formación Sierra de Animas, aproximadamente 55 Ma más joven que la de Sierra de Ríos, las rocas de alta sílice se asocian a rocas traquitoides con afinidades shoshoníticas. La aparición de vulcanitas con tendencias alcalinas parece indicar que la extensión se manifestaba a través de un sistema de fracturas profundas que se considera corresponde a ambiente de intraplaca y en el cual se registraban procesos de transcurrencia y de rift. Esto puede ser consecuencia de la progresiva cratonización de esta región con el incremento de la rigidez de la corteza.

Agradecimientos Para la realización de este trabajo ha sido valiosa la colaboración del CONICET (Buenos Aires,Argentina) y fundamental el apoyo de la Facultad de Agronomía de la Universidad de la República (Montevideo, Uruguay), quienes brindaron los medios materiales para los trabajos de campo y gabinete. Al ingeniero Lorenzo Ferrando quien contribuyó especialmente a mejorar nuestro conocimiento sobre la geología de la región de Aceguá y al que estamos reconocidos por sus valiosos comentarios y discusiones. La Sra. C. Olveira se encargó de la recopilación cartográfica. La lie. R. Navarro contribuyó con información inédita de localidades señaladas en la figura 1. Finalmente, nuestro agradecimiento a los Drs. W. Teixeira y K. Kawashita, del Centro de Pesquisas Geocronológicas (CPGeo), Universidad de Sao Paulo, Brasil, por su colaboración y apoyo en la obtención de datos geocronológicos.

\section{REFERENCIAS BIBLIOGRÁFICAS}

BOSSI, J. 1966. Geología del Uruguay. Montevideo, UDELAR. $464 \mathrm{p}$.

BOSSI, J. \& CAMPAL, N. 1992. Magmatisrao y tectónica transcurrente durante el Paleozoico Inferior en Uruguay. In: GUTIÉRREZ MARCO, J.C. ed. Paleozoico Inferior de Ibero-América. Mérida, Universidad de Extremadura, p. 343-356.

BOSSI, J. \& FERNANDEZ, A. 1963. Evidencias de diferenciación magmática hacia el final del Gondwana uruguayo. Bol. Paraná. Geociénc., 9:1-20.

BOSSI, J. \& NAVARRO, R. 1991. Geología del Uruguay. Montevideo, UDELAR. $966 \mathrm{p}$.

CORDANI, U.G. \& SOLIANI, E. 1990. Idades K-Ar e Rb-Sr das "Ilhas Cristalinas" de Rivera e Aceguá (Uruguai e Rio Grande do Sul, Brasil) e seu enquadramento no contexto geotectónico regional. $A n$. Acad. bras. Ci., 62(2): 145-156.

DALLA SALDA, L.; BOSSI, J.; CINGOLANI, C. 1988. The Rio de la Plata cratonic región of south-western gondwanaland. Episodes, 11(4):263-269.

ELIZALDE, G.; EUGUI, W.; VERDESIO, J.; STAPFF, M.; TELECHEA, J. 1970. Carta Geológica del Uruguay a Escala 1:100.000. Montevideo, UDELAR. 126 p. (Segmento Aceguá 3, Sector XXX)

FRAGOSO-CESAR, A.R.S.; WERNICK, E.; SOLIANI, E., Jr. 1982. Evolufáo geotectónica do Cinturáo Dom Feliciano. Urna contribucáo através da aplicacáo do modelo da tectónica de placas. In: CONGR. BRAS. GEOL., 32. Salvador, 1982. Anais... Salvador, SBG. v. 1, p. 13-23.

FRAGOSO-CESAR, A.R.S.; MACHADO, R : GÓMEZ RIFAS, C. 1987. Observa95es sobre o Cinturáo Dom Feliciano no Escudo Uruguaio e correlacóes com o Escudo do Rio Grande do Sul. In: SIMP. SUL-BRAS. GEOL., 3. Curitiba, 1987. Atas... Curitiba, SBG. bol. 2, p. 791-809.

HALPERN, M · UMPIERRE, M · URQUART, M · LINARES, E. 1972. Radiometric ages of crystalline rocks of southern South America as related to Gondwana and the Andean geologic provinces. In: UPPER MANTLE SIMPOSIUM. Buenos Aires, 1972. Proceedings... Buenos Aires, Comité Argentino de Manto Superior. v. 2, p. 345-356.

HART, S. 1966. Radiometric ages in Uruguay and Argentina and their implications concerning continental drift. Geol. Soc. Am. Meet. p. $62-65$.

HORBACH R.; KUCH, L; MARIMON, R.G.; MOREIRA, H.L.; FUCK, G.H.; MARIMON, M.P.C.; PIRES, J.H.; VIVIANI, O.; MARIANO, D.A.; TEIXEIRA, W. 1986. FolhaSH. 22-Porto Alegre e Parte das Folhas SH.21 - Uruguaiana - e 57.22 - Lagoa Mirim. Rio de Janeiro, IBGE p. 29-312.

LE MAITRE, R.W. 1978. Some problema of the projection of chemical data in mineralogical clasifications. Contrib. Mineral. Petrol., 56:186-189.
LE MAITRE, R.W. 1989. A Classification of Igneous Rocks and Glossary ofTerms. Oxford, Blackwell. $193 \mathrm{p}$.

NARDI, L.V.S.; LIMA, E.F.; GASTAL, M.C. 1992. O magmatismo no Grupo Bom Jardim: afinidade geoquímica e significado geotectónico. In: WORKSHOP BACIAS MOLASSICAS BRASILIANAS, 1. Local, 1992. Boletim Especial... Porto Alegre, p. 82-89.

PATINNO-DOUCE, A.E. 1992. Calculated relationships between activity of alumina and phase assemblages of silica-saturated igneous rocks. Petrogenetic implications of magmatic cordierite, garnet and aluminosilicate. J. Volcanol. Geotherm. Res., 52:43-63.

PECCERILLO, A. \& TAYLOR, S.R. 1976. Geochemistry of Eocene calcalkaline volcanic rocks from the Kastamonu área, northem Turkey. Contrib. Mineral. Petrol, 58:63-81.

PRECIOZZI, E; PENA, S.; MASQUELIN, E.; OYHANTCABAL, P. 1991. Memoria Explicativa del Fotoplano Piriápolis, Escala 1:100.000. Montevideo, DI.NA.MI,GE-UDELAR.

PRECIOZZI, F.; MASQUELIN, H.; SÁNCHEZ, L. 1993. Geología de la porción sur del Cinturón Cuchilla Dionisio. In: SIMP. INTERN. NEOPROTEROZOICO-CAMBRICO DE LA CUENCA DEL PLATA, 1. Las Palomas-Minas, 1993. Guia de Excursiones... Las Palomas-Minas, Uruguay, DI.NA.MI.GE. p. 3-39.

SOLIANI, E., Jr.; FRAGOSO CESAR, A.R.S.; TEIXEIRA, W.; KAWASHITA, K. 1984. Panorama geocronológico da porcáo meridional do Escudo Attântico. In: CONGR. BRAS. GEOL., 33. Rio de Janeiro, 1984. Anais... Rio de Janeiro, SBG. v. 2, p. $436-449$.

SOMMER, C.A.; FERNANDES DE LIMA, E.; NARDI, L.V.S. 1993. O vulcanismo ácido alcalino no Plato do Taquarembó, Dom Pedrito, R.S. In: SIMP. INTERN. NEOPROTEROZOICOCAMBRICO DE LA CUENCA DEL PLATA, 1. Las Palomas-Minas, 1993. Boletim de Resumas Expandidos... Las Palomas-Minas, Uruguay, DI.NA.MI.GE. Tomo 2, resumo $n^{9} 40$.

UMPIERRE, M. 1966. Datos geocronológicos de la Formación Sierra de Animas. In: BOSSI, J. Geologia del Uruguay. Montevideo, UDELAR. p. 134-135.

UMPIERRE, M. \& HALPERN, M. 1971. Edades Sr-Rb en rocas cristalinas del sur de la República Oriental del Uruguay. Asoc. Geol. Argent. Rev., 26(2):133-151.

WALTHER, K. 1927. Consideraciones sobre los Restos de un Elemento Estructural, aún Desconocido del Uruguay y el Brasil más Meridional. Montevideo, IGU - Imp. Nac. 47 p. (Boletín 9).

WILLIAMSON, J. 1968. Least-squares fitting of a straight Une. Can. J. Phys., 46:1845-1847.
MANUSCRITO A793

Recebidoem 13 de setembro de 1993 Revisáo do autor em 28 de julho de 1994 Revisáo aceita em 31 de agosto de 1994 